\title{
A Joint Power Allocation and Adaptive Channel Coding Scheme for Image Transmission over Wireless Channels
}

\author{
Mohamed El-Tarhuni \\ American University of Sharjah \\ Sharjah, UAE \\ mtarhuni@aus.edu
}

\author{
Mohamed Hassan \\ American University of Sharjah \\ Sharjah, UAE \\ mshassan@aus.edu
}

\author{
Akram Bin Sediq \\ Carleton University \\ Ottawa, Canada \\ akram@ @sce.carleton.ca
}

\begin{abstract}
In this paper, a joint adaptive power allocation and channel coding optimization scheme is proposed. This scheme exploits the difference in importance among bits used to represent an image or video signal. An offline iterative algorithm is developed to find the optimum combination of coding and power to be used for the transmission of individual bits. Optimality here is in the sense of minimizing the mean square error (MSE) which results in a better quality of the reconstructed image. Simulation results show that bits of significant importance should always be coded and allocated most of the transmitted power while bits of less significance may be sent without coding and with less allocated power. This is done while maintaining the average per-bit energy at the same level. Simulation results also show that the proposed combined approach achieves a gain of about $3 \mathrm{~dB}$ when compared to the case of coding alone. In addition, the proposed scheme outperforms the case of power allocation alone while reducing the peak-to-average power ratio.
\end{abstract}

\section{Index Terms}

Image transmission, wireless channels, MSE, power allocation.

\section{INTRODUCTION}

There has been a great interest in developing new wireless communication systems capable of not only sending voice signals but also images and video over mobile radio wireless links [1], [2]. This is to meet the ever increasing demand for multimedia services fueled by the surge in Internet utilization and the need for accessing different types of information while on the move. However, sending images and video signals require a communication system capable of sending at high data rates in the order of few Mbps, with low bit error probability. This is deemed to be very challenging due to the harsh nature of the wireless channels caused by multipath propagation and mobility of users. To meet these requirements, wireless communication systems that efficiently utilize the system resources are sought. These resources include transmission bandwidth and transmitted power.

Several studies have addressed the topic of resource allocation in wireless communication systems to overcome the time varying nature of wireless channels. An effective approach that improves the quality of signal transmission over wireless channels is channel coding which is typically considered as a main component of any wireless digital communications system. However, there is an increase in the required bandwidth (or equivalently reduction in the data rate) due to channel coding. Thus, it 
is always desired to minimize the overhead incurred by the channel coding scheme while meeting the applications' requirements in terms of the bit error rate (BER). A common problem with channel coding schemes is their degraded performance at low values of the received signal-to-noise ratio (SNR) and the unnecessary overhead when designed for worst case SNR values. To overcome this problem, adaptive channel coding schemes have been proposed to take advantage of knowledge of the channel status and/or the information being transmitted [3], [4], [5]. In some systems, like second generation GSM mobile radio systems, channel coding is used selectively for message bits that carry more information while no coding is used for less important bits [6]. A variation of the channel coding approach is joint source/channel coding (JSCC) [7], [8], [9], [10], [11], [12], [13], [14], [15]. In contrast to Shannon's information separation theorem [16], schemes employing JSCC jointly tradeoff source and channel coding to achieve significant performance improvements over separate source and channel coding. For example, the authors in [17] studied the problem of still image transmission over time-varying channels using channel state information (CSI) and wavelet image decomposition. They used a multi-resolution framework for both source coding and transmission via novel multi-resolution modulation constellations. In [18] an adaptive OFDM channel-condition based image transmission system is proposed. This system progressively transmits an image by allocating the modulation bit of each subcarrier based on the channel state. The authors in [19] proposed an OFDM joint modulation and source coding image transmission system.

Power control is another effective approach for mitigating the effect of fading on the quality of signal transmission over wireless channels [20], [21]. Systems adopting power control typically involve a mechanism for measuring the quality of the channel as seen by the receiver and providing such information to the transmitter to accordingly adjust the amount of transmitted power. For instance, if the channel is good then less power could be used while if the channel is bad then more power should be used. Few modifications to this strategy have been proposed. For instance, send higher data rates rather than reducing the power if the channel is good or even not to send at all if the channel is bad. These systems are classified as opportunistic systems since they take advantage of the information about the channel to optimize the communication process. The main issue with such systems is the need for a feedback link that is fast and reliable enough to track the time variation of the wireless channel without the need for the message structure of the transmitted image or video signal when allocating power. It has also been shown that the capacity of wireless channels can be achieved through other opportunistic methods like channel adaptive resource assignment techniques. For example, power control can mitigate the effect of fading on the quality of signal transmission over wireless channels [20] while channel-adaptive transmission rate combined with power assignment can achieve the capacity of wireless channels [22].

Recently, in [23], we proposed a novel algorithm that allocates power to information bits according to their importance. It was shown that the proposed algorithm was able to provide a signal-to-noise ratio gain of 3 to $4 \mathrm{~dB}$ for image transmission over Rayleigh fading channel without any increase in the bandwidth. The proposed scheme adjusts the amount of per-bit transmitted power according to the importance of that bit in terms of its impact on the quality of the image as measured by the mean square error (MSE). However, this scheme suffered from an increase in the peak-to-average power ratio (PAPR).

In this paper, we demonstrate the efficacy of the joint optimization of power allocation and adaptive channel coding for transmission of sensitive information over wireless channels. We consider the case of uncompressed images. While this might 
look a simplified assumption, it is worth noting that ceratin applications require that transmitted and received data should be entirely identical [24]. For instance, in telemedicine, digitized information such as CT images should not be changed during the transmission process, making diagnosis based on transmitted images as truthful as that based on original images. Any losses of image information during transmission might cause degradation in the quality of medical images. This in turn will affect the accuracy of the diagnosis. It is also worth noting that physicians involved in telemedicine are liable for their diagnosis based on transmitted images. This is another reason why differences in image quality before and after transmission should be minimal. In a future work we will study the impact of joint optimization of variable modulation and adaptive power allocation on the transmission of compressed images.

We propose a new algorithm that adaptively allocate power to the bitstream and decide on the level of needed channel coding to improve the image quality without increasing the PAPR significantly. The motivation is to exploit the advantages of both schemes while minimizing their drawbacks. For instance, while coding does not perform well at low SNR values, power allocation provides considerable gain improvements at low SNR values. Moreover, coding will help in decreasing the peak-to-average power ratio. The combined scheme is well suited for transmission of image and video signals, where different bits carry different amount of information. The combined scheme is specifically optimized for minimizing the MSE of the reconstructed image or video signal rather than the BER since it is more indicative of the image quality, as was shown in [23].

The rest of the paper is organized as follows. Section II briefly discusses related work on image transmission using power allocation and/or channel coding. In section III we present the system model. The proposed combined power optimization and coding algorithm is presented in section IV. Performance evaluation of this scheme is given in section V. Finally, section VI summarizes the results of the study and outlines our future work.

\section{RELATED WORK}

In this section we briefly summarize some of the related work. The authors in [25] proposed an unequal power allocation scheme for image transmission over multiple-input multiple-output (MIMO) systems. In their scheme, they divided the image into different quality layers to be simultaneously transmitted over different antennas using unequal power while having a constraint on the overall transmitted power during any symbol period. They targeted the minimization of the distortion in the transmitted images and argued that their scheme significantly improved the image quality when compared to conventional equal power allocations schemes. They also argued that this improvement was possible because the source statistics were considered when designing their transmission schemes without imposing any penalty on resources. In [26], the authors considered a similar problem to that in [25]. They also targeted the efficient transmission of JPEG images over MIMO systems using spatial multiplexing and multiple antennas to improve the data-rate, reliability, and throughput. They have also divided the image into different quality layers. Then, the antenna path with highest signal to interference noise ratio (SINR) is selected to transmit the most important layers of the image using an unequal power allocation scheme. An approach that minimizes the total-systemenergy of a wireless image transmission system is proposed in [27]. In [27], the authors presented an energy-optimized indoor wireless image transmission system that exploits the variabilities in the image information and the multi-path wireless channel. 
They characterized the variability in the image data by a rate-distortion curve while the variability in the wireless channel was characterized by its impulse response and its path-loss characteristics. In their proposed approach, they employed a dynamic algorithm that was utilized to obtain energy-optimal configurations subject to end-to-end performance constraints. In [28], the authors proposed two schemes for image transmission in wireless sensor networks (WSNs). The proposed schemes are energy-efficient open and closed loop schemes suitable for WSNs. The authors argued that the two schemes achieve energy conservation because they are wavelet-based such that images could be decomposed into multiple levels of resolution and different priorities. This in turn enables priority-based discarding of packets at the intermediate sensor nodes based on their battery's state-of-charge which also allowed a graceful trade-off of image quality and the sensor nodes' lifetime. The authors also analytically compared the open and closed loop schemes in terms of the dissipated energy. In [29], a position-oriented resource allocation scheme is proposed for image transmission in WSNs. The proposed scheme tries to optimize the quality of transmitted images under strict energy budget constraint. The proposed scheme is an image-pixel-position resource allocation scheme that works by exploring the unequal importance levels in the image bitstream. The authors in [30] proposed a cross-layer approach that selectively optimizes the quality of encrypted images when transmitted in WSNs with strict energy constraint. They proposed an unequal error protection that exploits the structure of image bitstreams such that resources are unequally and optimally allocated. The work in [31] proposed a statistical model to estimate the distortion in the transmission of progressive JPEG images due to quantization and channel bit errors. The proposed model included the different components of an entropy coder of lossy compression algorithms. Namely, it included compression techniques such as Huffman coding, differential pulse coding modulation (DPCM), and run-length coding. The authors argued that the proposed model could be used in designing efficient JSCC techniques for the transmission process independently of the image being transmitted and without the need to repeat the optimization procedures for the varying channel conditions. They also argued that this was possible due that the fact that their model can predict the average distortion over a diverse set of images. The authors presented an unequal power allocation image transmission scheme over Rayleigh flat fading channels as an application of their model. In this model they divided the image into different layers that were transmitted using unequal power while the total transmitted power over the length of the symbol stream was kept constant. Their results showed a PSNR gain in the order of 6 to5 dB at low SNRs when compared to the case of equal power allocation. In [32] and adaptive modulation technique was employed to improve the spectral efficiency when images are transmitted under the assumption of known channel state information (CSI) and a constrained retransmission request. The modulation level is adaptively selected based on the statistical or instantaneous CSI at the transmitter while the constrained retransmission includes one round-trip feedback of retransmission request and multiple retransmission response per request. Through their performance analysis and numerical results, the authors argued that CSI at the transmitter is very important for adaptive modulation and coding over fading channels.

\section{System Description AND Signal Model}

Figure 1 depicts the block diagram that characterizes the proposed scheme. Initially, the image or video signal is converted from its analog format into a digital format, i.e., the signal is sampled, quantized, and coded into $M$ bits per sample (pixel) 


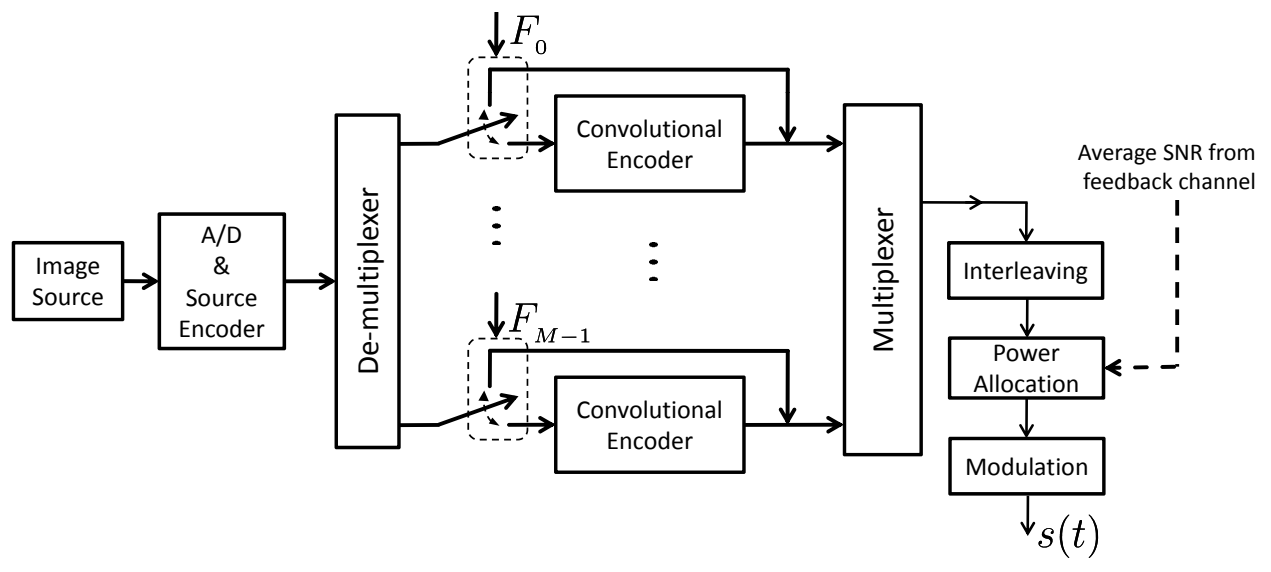

Fig. 1. Block diagram of the proposed coding-power allocation scheme.

where in this context one sample represents one pixel. The modulation scheme considered in this paper is a binary phase shift keying (BPSK) digital communication system. In this system, the bit stream is first processed by the coding block then by the power allocation algorithm. In more details, the bit stream will be divided into frames of size $N$ bits. Typical frame duration is $20 \mathrm{~ms}$ as used in second and third generation mobile radio standards, however smaller values could be used to reduce the encoding delay. Frames are then de-multiplexed into parallel streams (recall that $M$ bits are used per pixel). Demultiplexing is carried in a way such that each of those $M$ parallel streams contains bits of similar importance. In other words, most significant bits of consecutive samples (pixels) are placed together, then the next most significant bits, and so on. Using the proposed algorithm (to be described in section III), each stream of bits will be either fed to the channel encoder or passed without encoding according to the control vector set according to the operating energy per bit-to-noise power spectral density $\left(E_{b} / N_{0}\right)$ of the system. Although convolution coding is used in this paper, other encoding schemes may be used. The $M$ outputs of the channel encoders are multiplexed together followed by interleaving to overcome the effect of channel fading. Interleaving is done such that all bits corresponding to the same pixel of the original image are gathered back again to insure that the channel conditions will be the same for all of them. It is worth noting that the interleaving block will not only improve the performance of the decoder but also allow the feasibility of power allocation; since we can assume that the channel conditions will stay the same for the same pixel and hence the value of $E_{b} / N_{0}$ used to set the power allocated to each bit is the same. This $E_{b} / N_{0}$ will be the input to the power allocation algorithm to adjust the energy for each symbol according to its importance as will be described later on.

The transmitted BPSK signal during a frame is represented as:

$$
s(t)=\sum_{k=0}^{M-1} \sum_{i=0}^{\frac{L}{R_{c k}}-1} \sqrt{w_{k}} c_{k i} g\left(t-[k M+i] T_{s}\right)
$$

where $w_{k}$ is the transmitted power for the symbols in the $k^{\text {th }}$ stream, $c_{k i}$ is either the $i^{\text {th }}$ code symbol or the $k^{\text {th }}$ data bit depending on whether coding is used or not, $g(t)$ is a rectangular shape pulse of the transmitted signal, $T_{s}$ is the symbol duration, $R_{c k}$ is either one or the code rate depending on whether coding is used or not, and $L=N / M$ is the number of bits 
per stream which is assumed to be integer valued.

The wireless channel is modeled as a flat Rayleigh fading channel with received signal given by:

$$
r(t)=\alpha s(t-\tau)+n(t)
$$

where $\alpha$ represents the complex channel coefficient with amplitude that follows the Rayleigh distribution and uniform phase over $[0,2 \pi)$ and $\tau$ is the propagation delay. The additive white Gaussian noise (AWGN) is represented by $n(t)$ with zero-mean and two sided power spectral density of $N_{0} / 2$.

The received signal is processed using a matched filter and convolutional decoding using the Viterbi algorithm to obtain the original information bits. Decoded bits are then regrouped so that bits belonging to one sample (pixel) are placed in their proper order. Finally, digital to analog conversion is used to reconstruct the original image. The quality of the system is measured be calculating the MSE between the reconstructed image and the original image.

Let us define the power vector as $\underline{w}=\left[w_{0}, w_{1}, \ldots, w_{M-1}\right]$. In conventional power allocation schemes, all bits carry the same amount of power regardless of their significance. Thus, $\underline{w}$ is simply a vector of all ones; i.e. $w_{i}=1$ for $i=0,1, \ldots, M-1$. Such allocation is shown to be suboptimal and we propose to optimize the amount of power allocated to each symbol, i.e. optimize $\underline{w}$, such that more power is allocated to the most important bits under the constraint that the average energy per bit is kept the same as that of schemes employing equal power allocation. Note that there is no increase in the transmission bandwidth of the transmitted signal due to the proposed power allocation scheme and the only possible increase is due to channel coding. However, the peak-to-average power ratio (PAPR) will be increased. In the following section we propose an iterative algorithm that adjusts the power vector while keeping the PAPR under a specified value such that there is no significant degradation in the efficiency of the power amplifier used to amplify the signal.

\section{JOINT CODING AND POWER ALLOCATION ALGORITHM}

A main objective when designing any communications system is to minimize the average probability of bit error during transmission. The rationale behind this objective is the fact that minimizing the probability of error typically results in better quality of the received signal. However, this is not always the case. For instance, in voice, image, and video transmission, what is more important is the quality of the message after it is reconstructed at the receiver side. Therefore, a better performance measure in such cases is the root-mean square error (RMSE) rather than the BER. This is simply because bits transmitted by the system do not carry the same amount of information about the message. As a result, it is important to establish a relationship between the RMSE and the BER for such applications.

For a system with $M$ bits per sample, there are $2^{M}$ different samples to be transmitted. The binary representation of sample $x_{j}$ is given by the $j^{\text {th }}$ row of the following $2^{M} \times M$ matrix, 


$$
H=\left[\begin{array}{ccccc}
0 & 0 & 0 & \ldots & 0 \\
0 & 0 & \vdots & \vdots & 1 \\
\vdots & \vdots & \ddots & \vdots & \vdots \\
1 & 1 & 1 & \ldots & 0 \\
1 & 1 & 1 & \ldots & 1
\end{array}\right]
$$

with elements $h_{j k}$. The mean square error (MSE) is given by:

$$
\mathrm{MSE}=\sum_{j=0}^{2^{M}-1}\left(x_{j}-\hat{x}_{j}\right)^{2} P\left(x_{j}\right)
$$

where $\hat{x}_{j}$ is the reconstructed estimate of the $j^{\text {th }}$ sample after detection of the $M$ bits and $P\left(x_{j}\right)$ is the a priori probability that the $j^{\text {th }}$ sample is transmitted. Without loss of generality, consider the transmission of a sample that has a decimal value of zero with the binary representation of all zero elements $\left(h_{j k}=0\right.$, where $\left.k=0, \ldots, M-1\right)$. The possible received $M$ bits sequence will be one of the other $2^{M}-1$ combinations. The probability that the $i^{\text {th }}$ sample with a decimal value $i$ is reconstructed is given by:

$$
P S_{i}=\prod_{k=0}^{M-1}\left[P_{k} \gamma_{i 0}(k)+\left(1-P_{k}\right) \bar{\gamma}_{i 0}(k)\right]
$$

where $P_{k}$ is the probability that the $k^{\text {th }}$ bit is in error and $\gamma_{i 0}(k)$ is:

$$
\gamma_{i 0}(k)=\left\{\begin{array}{cc}
0 & \text { if } h_{0 k}=h_{i k} \\
1 & \text { if } h_{0 k} \neq h_{i k}
\end{array}\right.
$$

The notation $\bar{\gamma}_{i 0}(k)$ represents the binary inversion of $\gamma_{i 0}(k)$.

The MSE for the case of zero-valued sample is calculated as:

$$
\operatorname{MSE}_{0}=\frac{1}{2^{M}-1} \sum_{j=1}^{2^{M}-1} i^{2} \prod_{k=0}^{M-1}\left[P_{k} \gamma_{i o}(k)+\left(1-P_{k}\right) \bar{\gamma}_{i o}(k)\right]
$$

The MSE for other samples can be obtained following a similar procedure and the average MSE can be calculated by averaging over all possible samples. It is possible to show that, on average, all MSE values are approximately the same and hence equation (7) will be average MSE.

When coding is not used, the probability that the $k^{\text {th }}$ bit is in error in AWGN is given by:

$$
P_{k}=Q\left(\sqrt{\frac{2 E_{b}(k)}{N_{0}}}\right)
$$


where $E_{b}(k)=w_{k} T_{b}$ is the energy of the $k^{\text {th }}$ bit, $T_{b}$ is the bit duration, and $Q($.$) is the Q-function. When convolutional coding$ is used, the probability that the $k^{\text {th }}$ bit is in error can be upper bounded by [33]:

$$
P_{k}<\sum_{d=d_{\text {free }}}^{\infty} \beta_{d} Q\left(\sqrt{2 \frac{E_{b}(k)}{N_{0}} R_{c} d}\right)
$$

where $d_{\text {free }}$ is the free distance for the convolution code and $\beta_{d}$ denotes the total input weight of error events at Hamming distance $d$. For flat Rayleigh fading channels, the probability of error for the BPSK system is given by [10]:

$$
P_{k}=\frac{1}{2}\left(1-\sqrt{\frac{\bar{\mu}(k)}{1+\bar{\mu}(k)}}\right)
$$

where $\bar{\mu}(k)=\frac{E_{b}(k)}{N_{0}} E\left\{|\alpha|^{2}\right\}$, which is the average signal-to-noise ratio for the $k^{\text {th }}$ bit.

\section{A. Power Allocation Algorithm}

The problem at hand can be stated as follows. We would like to find the optimum power distribution such that the RMSE is minimized subject to the constraints that the average energy per bit is kept constant and the PAPR is kept below a certain limit. The above optimization is done for RMSE regardless of the obtained average probability of bit error. The proposed iterative power allocation algorithm is as follows:

\section{Initialization:}

i. The power distribution vector $\underline{w}$ is initialized to all ones (assume the energy is the same for all bits with $w_{i}=1$ for $i=0,1, \ldots, M-1$.

ii. The coding flag vector, $F$, is initialized to all zeros (assuming that no coding will be done for any of the bits, i.e. $F_{i}=0$ for $i=0,1, \ldots, M-1$.

iii. The MSE is calculated using (7) and (8) for a given $E_{b} / N_{0}$.

iv. Define two bits, $B$ is borrowing power and $D$ is donating power.

v. The maximum limit of the PAPR is set to PAPR $\max$ and the energy step size is set to $\Delta E_{b}$.

\section{Iteration:}

i. Starting with $B=M-1$, most significant bit (MSB) as borrower; and $D=0$ (LSB as donor).

ii. The energy for the $D$ bit is reduced by $\Delta E_{b}$ and the energy of $B$ bit is increased by the same amount such that during the $n^{\text {th }}$ iteration we have

$$
\begin{aligned}
& E_{b D}(n)=E_{b D}(n-1)-\Delta E_{b} \\
& E_{b B}(n)=E_{b B}(n-1)+\Delta E_{b}
\end{aligned}
$$

Note that within a block of $M$ bits, the minimum energy per bit is zero and the maximum energy per bit is $M E_{b}$ where $E_{b}$ is the average energy per bit.

iii. The MSE by is re-calculated using (7) and (8) for the uncoded bits and using (7) and (9) for 
the coded bits for the following cases (note that the coding flag vector determines the bits to be coded).

(a) Both the borrower and the donor are coded.

(b) The borrower is coded while the donor is not.

(c) The donor is coded while the borrower is not.

(d) Both of the borrower and the donor are not coded.

According to the previously calculated minimum value of the MSE, the coding flag vector is updated such that:

(a) $F_{B}=1$ and $F_{D}=1$ if case (a) gave the minimum MSE.

(b) $F_{B}=1$ and $F_{D}=0$ if case (b) gave the minimum MSE.

(c) $F_{B}=0$ and $F_{D}=1$ if case (c) gave the minimum MSE.

(d) $F_{B}=0$ and $F_{D}=0$ if case (d) gave the minimum MSE.

The energy of the two bits is changed as explained above until the minimum value of MSE is obtained

while the PAPR is kept less than $\mathrm{PAPR}_{\max }$.

iv. The same procedure outlined above in (ii) and (iii) is repeated but with the donor bit incremented by one until all least significant bits $D$ are used.

v. Next, the borrower bit is decreased by one to optimize the second most significant bit $(B=N-2)$ and steps

(ii) till (iv) are repeated.

vi. The above steps are repeated until all bits are optimized; i.e. $B=0$.

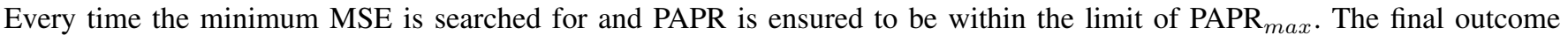
of this algorithm is a lookup table that specifies for every the amount of power to be allocated for a particular bit stream. It also decides whether coding should be used or not.

Table 1 shows the distribution of the flag vector $F$ for different values of $E_{b} / N_{0}$ under AWGN. A value of " 0 " means no coding is used for that particular stream while a value of "1" means coding should be applied. As can be seen in table 1 , when $E_{b} / N_{0}$ is less than $4 \mathrm{~dB}$, the least significant bits are not coded while the most significant bits are coded. On the other hand, for high $E_{b} / N_{0}$, all bits need to be coded in order to minimize the MSE. The optimum power weight for each bit is shown in Figure 2 for both the power allocation alone and the joint power allocation and coding schemes. Bit 7 is the most significant bit while bit 0 is the least significant bit. It is observed that, for small values of $E_{b} / N_{0}$, most of the power should be allocated to the most significant bits $(7,6$, and 5). As the signal-to-noise ratio increases, least significant bits start to play a role on the image quality and should be allocated more power. Eventually, for high $E_{b} / N_{0}$ values, the power distribution would reach the conventional equal power scheme as previously noticed. We also remark that the use of coding along with power allocation has resulted in a reduction in the range of the power coefficients when compared to the power allocation alone. This results in a reduction in the PAPR as supported by the results presented later in Figure 6.

It is important to note that the above optimization scheme is done only once for each $E_{b} / N_{0}$. In other words, this algorithm does not require real time processing of the data and is carried offline only once. After the power coefficients are obtained, 
TABLE I

CODING FLAG: "0" THE CORRESPONDING BIT IS NOT CODED; “0” THE CORRESPONDING BIT IS CODED $(M=8)$

\begin{tabular}{|c|c|c|c|c|c|c|c|c|}
\hline $\begin{array}{c}E_{b} / N_{0} \\
(\mathrm{~dB})\end{array}$ & $\begin{array}{c}\text { LSB } \\
0\end{array}$ & & & & & & & $\begin{array}{c}\text { MSB } \\
M-1\end{array}$ \\
\hline 0 & 0 & 0 & 0 & 0 & 1 & 1 & 1 & 1 \\
\hline 1 & 0 & 0 & 0 & 1 & 1 & 1 & 1 & 1 \\
\hline 2 & 0 & 0 & 1 & 1 & 1 & 1 & 1 & 1 \\
\hline 3 & 0 & 1 & 1 & 1 & 1 & 1 & 1 & 1 \\
\hline 4 & 0 & 1 & 1 & 1 & 1 & 1 & 1 & 1 \\
\hline 5 & 1 & 1 & 1 & 1 & 1 & 1 & 1 & 1 \\
\hline 6 & 1 & 1 & 1 & 1 & 1 & 1 & 1 & 1 \\
\hline 7 & 1 & 1 & 1 & 1 & 1 & 1 & 1 & 1 \\
\hline 8 & 1 & 1 & 1 & 1 & 1 & 1 & 1 & 1 \\
\hline 9 & 1 & 1 & 1 & 1 & 1 & 1 & 1 & 1 \\
\hline 10 & 1 & 1 & 1 & 1 & 1 & 1 & 1 & 1 \\
\hline
\end{tabular}

all what the transmitter needs is an estimate of $E_{b} / N_{0}$ and it can look up the table to decide which bits to be coded and how much power should be allocated to them. When $E_{b} / N_{0}$ changes, the transmitter uses the same table to look up the appropriate combination of coding and power coefficients without the need of repeating the optimization process. Thus, this algorithm does not add much complexity to conventional systems.
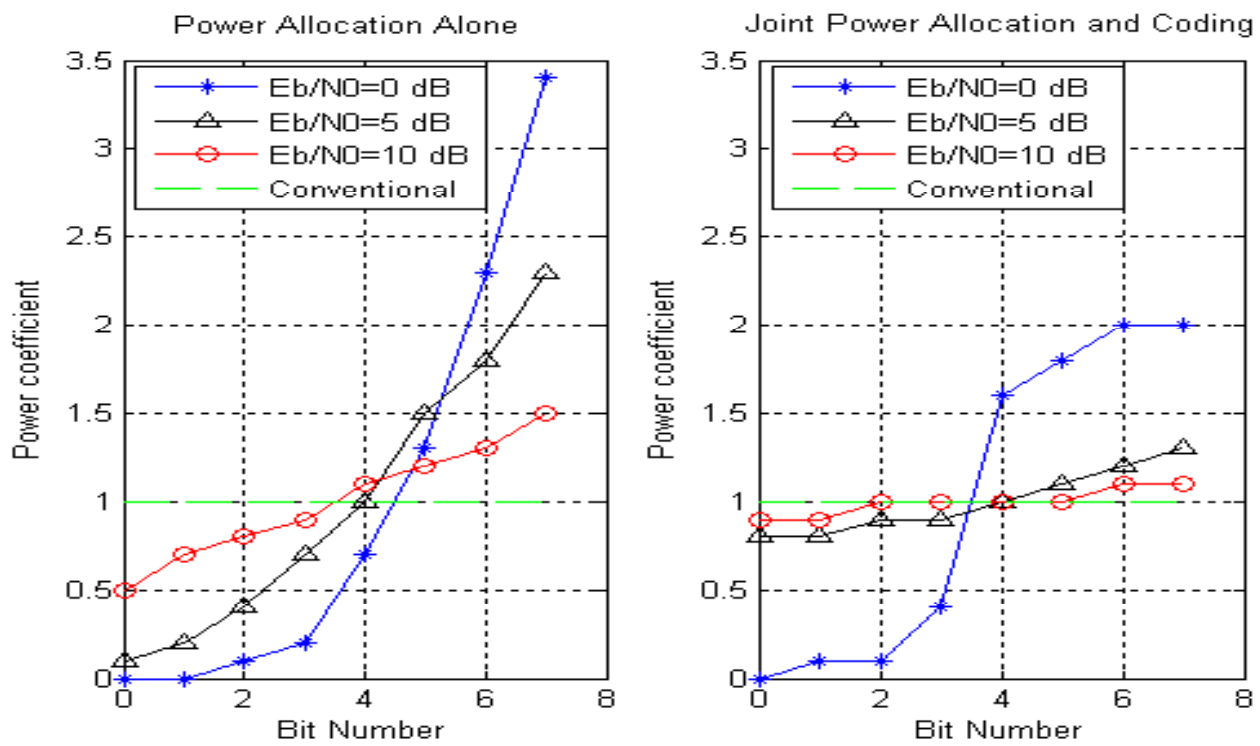

Fig. 2. Power allocation for different values of $E_{b} / N_{0}$.

\section{Simulations AND NUMERICAL RESUlts}

In this section, we study via simulations the performance of the proposed scheme. The RMSE is obtained using different settings to evaluate the efficacy of the proposed scheme. More specifically, we obtain the RMSE for the following cases; a conventional equal-power scheme is used, a scheme that employs power allocation alone, a scheme that employs coding alone, and finally the proposed joint power allocation and adaptive coding scheme. We also consider the cases of AWGN channel along with a fading case. The following results are for an image of size of 537 by 358 pixels where each pixel is represented 
by eight bits. When coding is used, a 1/2 rate convolutional code is used at the transmitter and Viterbi decoding is used at the receiver.

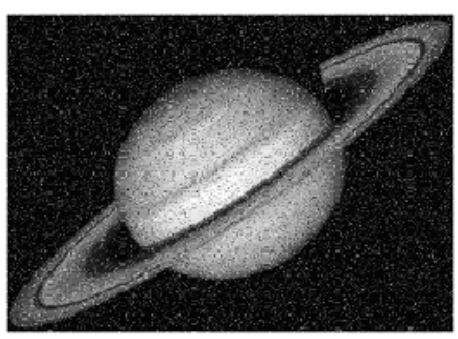

(a) conventional

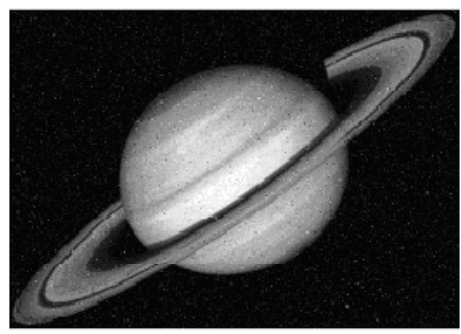

(c) power allocation alone

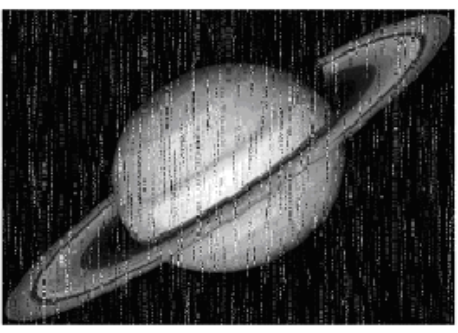

(b) coding alone

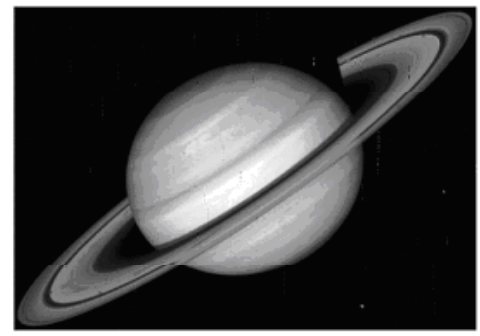

(d) combined technique

Fig. 3. Comparison of image transmission performance over AWGN at $E_{b} / N_{0}=1 \mathrm{~dB}$.

Figure 3 visually depicts the performance of the abovementioned four different schemes over an AWGN channel. It is obvious that the scheme employing coding alone does not provide a good picture quality when compared to the scheme employing power allocation alone. This actually is expected since both schemes are operating at a relatively low $E_{b} / N_{0}$ of $1 \mathrm{~dB}$ and coding tends to cause more errors. However, for the same low value of $E_{b} / N_{0}$, the proposed combined adaptive coding and power allocation schemes outperforms all other schemes and provides superior image quality.

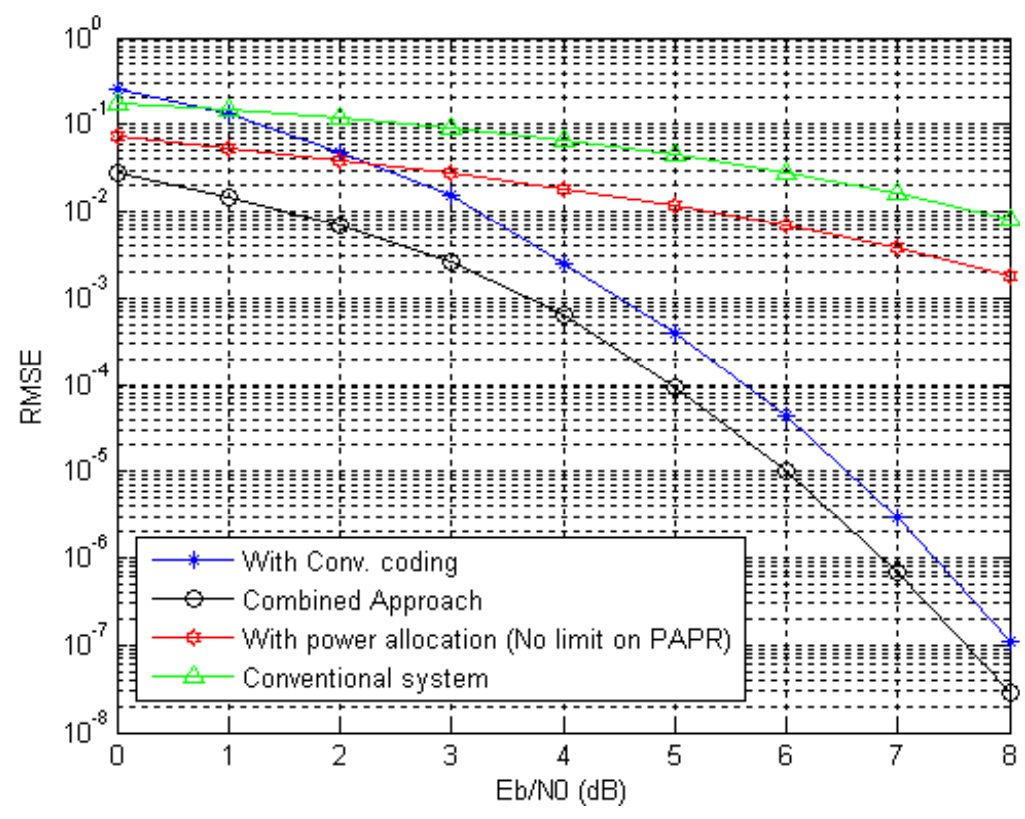

Fig. 4. RMSE performance for image transmission in AWGN with no PAPR limits. 


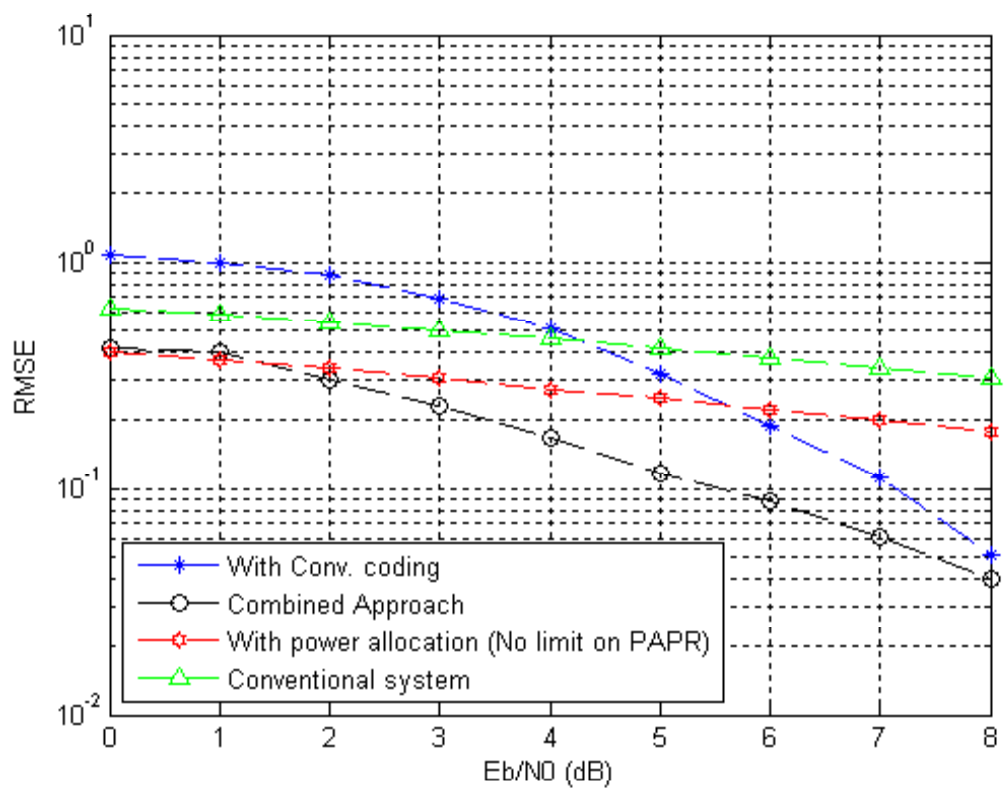

Fig. 5. RMSE performance for image transmission in flat Rayleigh fading with no PAPR limits.

To have a quantitative comparison, Figure 4 shows the RMSE as a function of $E_{b} / N_{0}$ for the four schemes in AWGN. There was no limit imposed on the PAPR, so this figure shows the ultimate gain that can be achieved with power allocation. As can be seen, coding alone is enough for high $E_{b} / N_{0}$ values but not enough for low $E_{b} / N_{0}$ values while power allocation alone is good for low $E_{b} / N_{0}$ values. The figure also shows that the proposed joint approach achieves excellent performance over the whole range of $E_{b} / N_{0}$ since it is always trying to optimize the performance according to the operating $E_{b} / N_{0}$. Specifically, it is clear that at low $E_{b} / N_{0}$, the proposed scheme outperforms the cases of coding alone or the power allocation alone by a gain of about 2 to $3 \mathrm{~dB}$.

Figure 5 shows the RMSE performances when a flat Rayleigh fading channel is encountered. Obviously, there is a clear degradation in performance due to fading when compared to the AWGN case. Nevertheless, the proposed combined scheme is superior to all other schemes over the entire range of $E_{b} / N_{0}$. It provides a signal-to-noise ratio gain of about $3 \mathrm{~dB}$ for $2 \leq E_{b} / N_{0}<7$. The gain is even higher when compared to conventional and power allocation alone schemes for high $E_{b} / N_{0}$. We remark that although other schemes like antenna diversity or wideband transmission could be used to mitigate the fading effect, the gain of the proposed scheme is expected to be sustained.

The maximum allowed PAPR value and the $E_{b} / N_{0}$ operating value affect the improvement in performance for the cases when power allocation alone is used as well as when the proposed joint coding and power allocation scheme is used. This effect can be clearly seen in Figure 6. It is obvious that more gain is achieved when the constraint in the PAPR is relaxed. It can also be noted that for both schemes the gain reduces as $E_{b} / N_{0}$ increases. This means that for high signal-to-noise ratios equal power allocation schemes would result in better performance. Note that most of the gain obtained for the case of power allocation alone is achieved with a PAPR of $4.5 \mathrm{~dB}$; while a similar behavior is obtained for PAPR values less than $2.5 \mathrm{~dB}$ for the proposed joint coding and power allocation scheme. Thus, the combined approach results in $2 \mathrm{~dB}$ reduction in the PAPR 


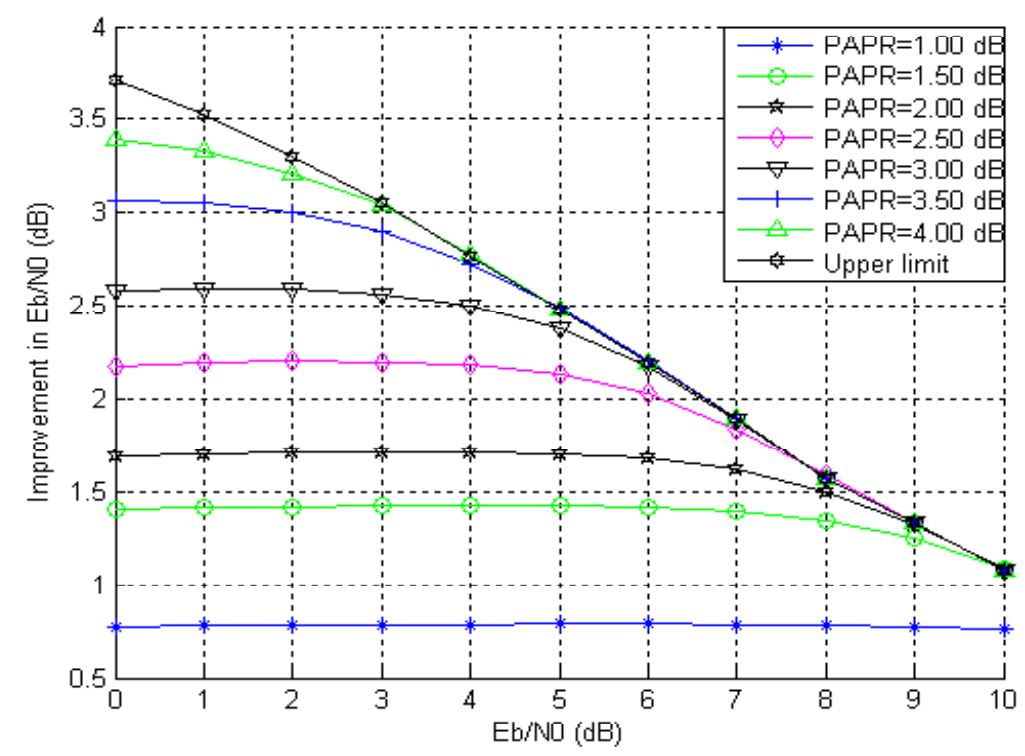

(a)

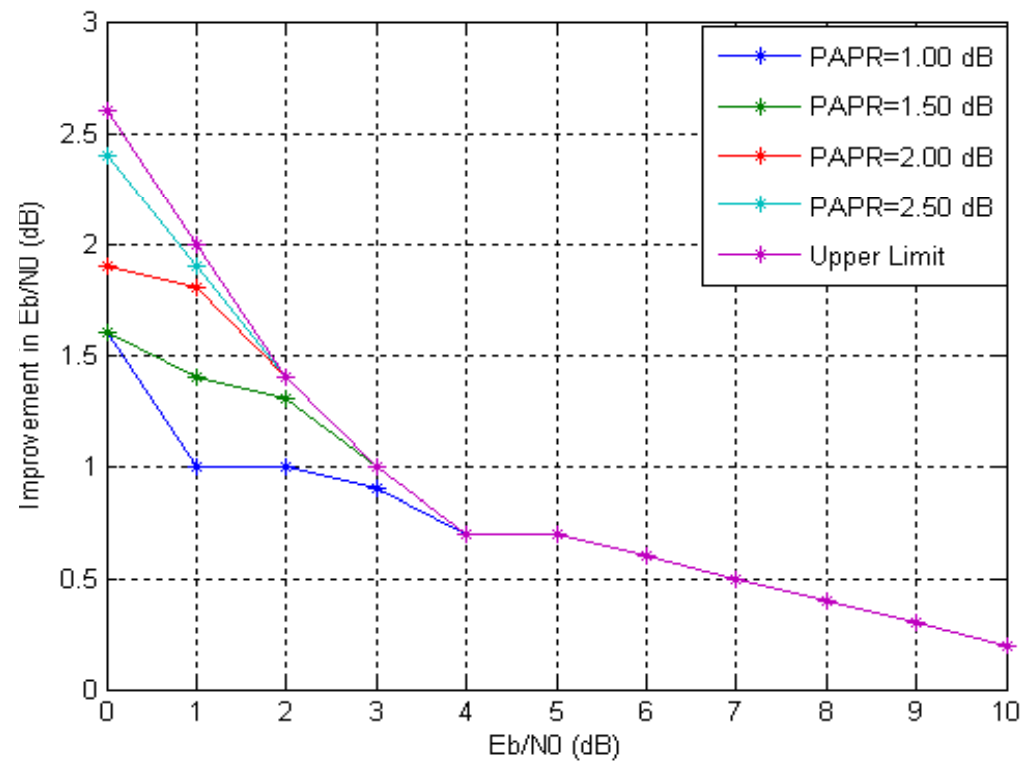

(b)

Fig. 6. Improvement in $E_{b} / N_{0}$ for different PAPR limits: (a) Power allocation alone relative to conventional scheme; (b) Combined scheme relative to conventional convolutional coded scheme.

and, hence, better power amplifier efficiency.

\section{CONCLUSIONS}

In this paper, we proposed a scheme that jointly optimize power allocation and channel coding to improve the quality of images and video sequences when transmitted over wireless channels. As opposed to conventional schemes that treat all bits equally in terms of power and coding, bits used to represent the image samples are treated differently according to their importance to the quality of the image. An iterative offline algorithm is proposed to find the optimum combination of power coefficients and coding for each bit. The minimum mean-square error (MSE) of the reconstructed image is used as 
the optimization criterion rather than the average probability of error. The presented results show that the mean-square error performance is significantly improved when compared to the case of coding alone or power allocation alone, with a gain of about $3 \mathrm{~dB}$ in $E_{b} / N_{0}$. While achieving this gain, the proposed scheme also reduces the peak-to-average power ratio in comparison to the case of power allocation alone with a typical reduction of about $2 \mathrm{~dB}$.

\section{REFERENCES}

[1] 3GPP2 C.S0002. Physical layer standard for CDMA2000 spread spectrum system. (www.3gpp2.org), June 2001.

[2] 3GPP. Technical specification group radio access network: physical layer-general specification. (www.3gpp.org), December 2003.

[3] L. Cao and C. Chen. Multiple hierarchical image transmission over wireless channels. Journal of Visual Communication and Image Representation, 13(3):386 - 399, 2002.

[4] ETSI. Digital video broadcasting (DVB); framing structure, channel coding and modulation for digital terrestrial television. (http://www.etsi.org/home.htm).

[5] N. Thomos, S. Argyropoulos, N. Boulgouris, and M. Strintzis. Robust transmission of H.264/AVC streams using adaptive group slicing and unequal error protection. EURASIP J. Appl. Signal Process., pages 120-120, 2006.

[6] Theodore Rappaport. Wireless communications: Principles and practice. Prentice Hall, Englewood Cliffs, NJ, 2002.

[7] M. E. Buckley, M. G. Ramos, S. S. Hemami, and S. B. Wicker. Perceptually-based robust image transmission over wireless channels. In Proceedings of The International Conference on Image Processing, 2000.

[8] Z. Wu, A. Bilgin, and M. Marcellin. Joint source/channel coding for image transmission with JPEG2000 over memoryless channels. IEEE Transactions on Image Processing, 14(8):1020-1032, Aug. 2005.

[9] J. Cai and C. Chen. Robust joint source-channel coding for image transmission over wireless channels. IEEE Transactions on Circuits and Systems for Video Technology, 10(6):962-966, Sept. 2000.

[10] R. H. Morelos-Zaragoza, M. P. C. Fossorier, S. Lin, and H. Imai. Multilevel coded modulation for unequal error protection and multistage decodingpart I:symmetric constellations. IEEE Transactions on Communications, 48(2):204-213, Feb. 2000.

[11] X. Wang and M. T. Orchard. Design of superposition coded modulation for unequal error protection. In Proceedings of the IEEE International Conference on Communications (ICC), pages 412-416, June 2001.

[12] X. Dong-liang and S. Hong. Joint source-channel decoding scheme for image transmission over wireless channel. Journal of Natural Sciences, 7(3):307-312, 2002

[13] M. Hassan and M. Krunz. Video streaming over wireless packet networks: An occupancy-based rate adaptation perspective. IEEE Transactions on Circuits and Systems for Video Technology, 17(8):1017-1027, August 2007.

[14] L. Atzori, M. Krunz, and M. Hassan. Cycle-based rate control for one-way and interactive video communications over wireless channels. IEEE Transactions on Multimedia, 9(1):176 -184, jan. 2007.

[15] M. Hassan and T. Landolsi. A retransmission-based scheme for video streaming over wireless channels. Wireless Communications and Mobile Computing, 10(4):511-521, 2010.

[16] T. Cover and J. Thomas. Elements of Information Theory. John Wiley \& Sons, New York, 1991.

[17] I. Kozintsev and K. Ramchandran. Robust image transmission over energy-constrained time-varying channels using multiresolution joint source-channel coding. IEEE Transactions on Signal Processing, 46(4):1012-1026, April 1998.

[18] H. Chu, B. Park, C. Koo An, J. Kang, and H. Son. Wireless image transmission based on adaptive OFDM system. In International Forum on Strategic Technology, 2007. IFOST 2007., pages $623-626$, oct. 2007.

[19] A. Doufexi, A. Nix, and D. Bull. Robust wireless image transmission using jointly-optimized modulation and source coding. In Proceedings of the IEEE VTC'00 Conference, pages 2039-2043, 2000.

[20] Q. Zhang, Z. Ji, W. Zhu, and Y. Zhang. Power-minimized bit allocation for video communication over wireless channels. IEEE Transactions on Circuits and Systems for Video Technology, 12(6):398-410, June 2002. 
[21] S. L. Kim, Z. Rosberg, and J. Zander. Combined power control and transmission rate selection in cellular networks. In Proceedings of the IEEE VTC'99 Conference, pages 1653-1657, Vienna, Austria, 1999.

[22] A. J. Goldsmith and S. Chu. Variable-rate variable-power M-QAM for fading channels. IEEE Transactions on Information Theory, 45(10):1218-1230, Aug. 1997.

[23] A. Bin Sediq and M. El Tarhuni. MMSE power allocation for image and video transmission over wireless channels. In Proceedings of The 16th IEEE International Symposium on Personal, Indoor and Mobile Radio Communications (PIMRC'05), 2005.

[24] C. Huang and S. Liang. An implementation of wireless medical image transmission system on mobile devices. Journal of Medical Systems, 32(6):471-480, 2008.

[25] M. Sabir, A. Bovik, and R. Heath. Unequal power allocation for JPEG transmission over MIMO systems. IEEE Transactions on Image Processing, 19(2):823-423, Feb. 2010.

[26] R. Deepa and K. Baskaran. MIMO based efficient jpeg image transmission and reception by multistage receivers. International Journal of Computer Applications, 1(15):13-23, 2010.

[27] S. Appadwedula, M. Goel, N. Shanbhag, D. Jones1, and K. Ramchandran. Total system energy minimization for wireless image transmission. The Journal of VLSI Signal Processing, 27(1-2):99-117, 2004.

[28] V. Lecuire, C. Duran-Faundez, and N. Krommenacker. Energy-efficient image transmission in sensor networks. International Journal of Sensor Networks, 4(1-2):37-47, 2008.

[29] W. Wang, D. Peng, H. Wang, H. Sharif, and H. Chen. Energy-constrained distortion reduction optimization for wavelet-based coded image transmission in wireless sensor networks. IEEE Transactions on Multimedia, 10(6):1169 -1180, oct. 2008.

[30] W. Wang, D. Peng, H. Wang, H. Sharif, and H. Chen. Energy-constrained quality optimization for secure image transmission in wireless sensor networks. Adv. MultiMedia, 2007(1):1-9, 2007.

[31] M. Sabir, R. Heath H. Sheikh, and A. Bovik. A joint source-channel distortion model for JPEG compressed images. IEEE Transactions on Image Processing, 15(6):1349-1364, Feb. 2006.

[32] K. Peng, J. Kieffer, Q. Liu, and S. Zhou. Embedded image transmission based on adaptive modulation and constrained retransmission over block fading channels. In Proceedings of the IEEE International Conference on Acoustics, Speech, and Signal Processing,ICASSP-93., volume 5, pages 696-699, April 2003.

[33] J. Proakis and M. Salehi. Digital Communications. McGraw-Hill, 5th edition, 2007. 